\title{
Attitude of the Hong Kong Small and Medium Enterprises (SMEs) towards Information Security
}

Hon Keung YAU*

Systems Engineering and Engineering Management, City University of Hong Kong, Kowloon Tong, Kowloon, Hong Kong

\section{Introduction}

Bayuk (2009) states that Information security policies are a special type of documented business rule to protect valuable data and systems which store and process the information [1]. Within an organization, these written policy documents provide a high -level description of the various controls the organization will use to protect information. The documented information security policy is also a formal declaration of management's intent to protect information, and it is required for compliance with various security and privacy regulations. Organizations that require audits of their internal systems for compliance with various regulations will often use information security policies as the reference for the audit.

\section{Attitude of the Hong Kong SMEs towards information security}

The following information is the summary of "e-Security Survey Report" conducted by Hong Kong Internet Service Providers Association in 2002 [2]. The sample size of this survey is 318 SMEs in Hong Kong. The statistics data can be used to explain about the attitude for the SMEs towards information security. $37 \%$ of respondents said that security is only somewhat important to their company. The other $37 \%$ SMEs said it's important and only $20 \%$ said that it's extremely important. 7.4\% SMEs even classified security as not important to their business operations. The survey results showed that over $83 \%$ of the SMEs employed Anti Virus security technologies. The second most popular security technologies that are used as the security gateway are password /log-in (68\%) and the third is firewall (39\%). It shows that HK SME main concern on information security placed on Anti

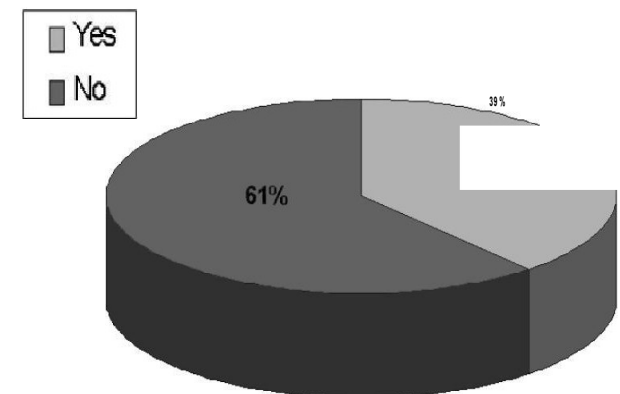

Figure 1: Is there any security Policy adopted (Source:Hong Kong Internet Service Providers Association 2002).

Virus. Only 39\% of the sample SMEs adopted information security policy in their company. This shows that HK SMEs did not employ comprehensive information security program. And the result may be due to the reason that it is difficult for them to sett security policies to match business goals (Figure 1).

\section{Conclusions}

Information security policy is important to all Hong Kong SMEs. Most Hong Kong SMEs adopted the information security policy and they mainly focused on anti-virus protection.

\section{References}

1. Bayuk J (2009) How to Write an Information Security Policy. Computer World.

2. Hong Kong Internet Service Providers Association (2002) Security survey.

*Corresponding author: Hon Keung YAU, Systems Engineering and Engineering Management, City University of Hong Kong, Kowloon Tong, Kowloon, Hong Kong, E-mail: honkyau@cityu.edu.hk

Received November 08, 2013; Accepted November 08, 2013; Published November 11,2013

Citation: Hon Keung YAU (2013) Attitude of the Hong Kong Small and Medium Enterprises (SMEs) towards Information Security. Adv Robot Autom 2: e114. doi: 10.4172/2168-9695.1000e114

Copyright: (c) 2013 Hon Keung YAU. This is an open-access article distributed under the terms of the Creative Commons Attribution License, which permits unrestricted use, distribution, and reproduction in any medium, provided the original author and source are credited. 